\title{
Characterization of the silver thin films produced at different substrate temperatures
}

\author{
Ton Nu Quynh Trang ${ }^{1}$, Le Thi Ngoc $\mathrm{Tu}^{2}$, Tran Van Man ${ }^{3}$, Vu Thi Hanh Thu ${ }^{1, *}$
}

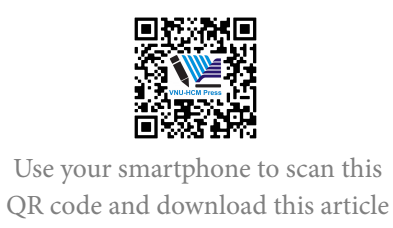

${ }^{1}$ Faculty of Physics and Engineering Physics, VNUHCM-University of Science

${ }^{2}$ Falculity of Physics, Dong Thap

University

${ }^{3}$ Faculty of Chemistry,

VNUHCM-University of Science

\section{Correspondence}

Vu Thi Hanh Thu, Faculty of Physics and Engineering Physics, VNUHCM-University of Science

Email: vththu@hcmus.edu.vn

History

- Received: 2019-06-30

- Accepted: 2019-11-14

- Published: 2019-12-31

DOI : 10.32508/stdj.v22i4.1691

\section{Check for updates}

\section{Copyright}

(c) VNU-HCM Press. This is an openaccess article distributed under the terms of the Creative Commons Attribution 4.0 International license.

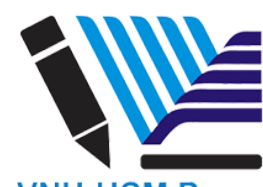

\begin{abstract}
Introduction: In recent decades, the antimicrobial surfaces/coating properties towards a longlasting microbicidal effect have drawn enormous attention by researchers, they have been developed and used in a wide variety of high-touch hospital devices as a potential approach. Methods: In this work, Ag NPs was synthesized by sputtering method th the different annealing temperatures of $100^{\circ} \mathrm{C}, 200^{\circ} \mathrm{C}, 300^{\circ} \mathrm{C}$, and $400^{\circ} \mathrm{C}$. Results: As a result, the as-synthesized Ag-300 exhibits the highest E. coli antibacterial performance compared with others. This can be attributed to the change of the Ag NPs toxicity based on the growth of nanoparticles during the deposition process related to the Ostwald ripening process, thermal activation and coalescence particles. Conclusion: This work provides an essential insight into the antimicrobial activity of Ag NPs-based films synthesized through the vacuum deposition technique, resulting in opening a new approach for enhancing the antimicrobial efficacy and prospects.
\end{abstract}

Key words: Silver nanoparticles, antibacterial mechanism, vacuum deposition techniques, toxicity

\section{INTRODUCTION}

Nanoparticles (NPs) have been considered as one of the most promising alternatives for traditional materials in many fields of science and technology ${ }^{1,2}$, due to nanoscale approaching physical characteristics and functionalities that can be assigned to the different from their bulk counterparts. As an example, the antibacterial performance of nanomaterials such as $\mathrm{ZnO}, \mathrm{TiO}_{2}$, and $\mathrm{Ag}$ NPs have drawn enormous attention in recent years for their desired applications in biomedical applications, water disinfection, and consumer goods ${ }^{3,4}$. However, a current drawback is the synthesis and application of nanoparticles to implement effective measures that can prevent wound infections burns and chronic ulcers associated with healthcare caused by microorganisms. Specifically, healthcare-associated infections have been considered as one of the global threats related to bacterial pathogen emergence and one of the main reasons for patient morbidity and mortality. Indeed, an approximated $20 \%$ to $40 \%$ of health associated with the infection fields was reported ${ }^{5}$. Among all nanomaterials, silver nanoparticles (Ag NPs) not only have attracted more attention in healthcare-associated fields but also considered as one of the most promising candidates for potential medical applications in recent years due to their unique nano-physicochemical characteristics and broad-spectrum antimicrobial activity ${ }^{6,7}$. Previous studies reported that Ag NPs, which synthesized in a similar way to form silver ions, exerted against bacteria through a multifactorial process, and they were associated with inhibiting the growth of harmful bacteria as they were harm to the bacterial cell wall and plasma membrane or restraint on DNA replication and protein ${ }^{8}$. They could be released by the natural formation of ion $\mathrm{Ag}$ in the presence of reductive components in the environment ${ }^{9}$.

Moreover, the characteristic features of nanoparticles such as size, shape, density distribution have been demonstrated to affect the antibacterial activity of $\mathrm{Ag}$ NPs significantly, this could be attributed to a differential release of $\mathrm{Ag}^{+}$ions ${ }^{10}$. Previous researches in this field suggested that the effects of shapes and sizes prepared by wet chemical reduction methods or biosynthesis play a significant role in the antimicrobial nature of Ag NPs ${ }^{11-14}$. These methods have been devoted to controlling the size and shape of the $\mathrm{Ag}$ NPs, however, still, some obstacles such as the presence of colloidal stabilizers or impurities, the toxic solvents, and the sophisticated synthesis process to reduce or suppress the aggregation phenomenon in solution were detected ${ }^{15-17}$. Good adhesion between the Ag NPs and the substrate has great potential in the practical applications, while, the mere synthesis of the Ag NPs is rather hard for most of the desired applications. Therefore, an explored alternative route has been centered on solving the aforementioned drawbacks of wet chemical methods. Amongst, vacuum 
deposition techniques consist of thermal evaporation, magnetron sputtering and pulsed laser deposition have drawn enormous attention in recent years and considered as an effective method to expand their applications because it can be grown on large surface areas with good quality, easy control in fabrication process, and environmentally friendly based on different preparation conditions. In which, the temperature factor has been considered as one of the most critical factors for a reaction, adhesion and phase separated morphology at the interface that can affect the change in morphology on flat substrates in the growth process of the thin film significantly. Compared with other Ag types (Ag suspension, powder...), Ag thin film on the flat substrates has some advantages i) they can save the material that can obtain the surface antibacterial performance equally; ii) the diffusion of Ag nanoparticles into the environment is limited due to the good adhesion of Ag nanoparticles on the substrates deposited via sputtering method.

In this work, Ag NP films have been prepared by DC magnetron sputtering directly on the surface of glass at the different annealing temperature from $100^{\circ} \mathrm{C}$ to $400^{\circ} \mathrm{C}$ to investigate the change of the crystalline structure, optical, morphology, and their antibacterial activity against various E. Coli bacteria. These results support the new approach for the design and synthesis of other precious metal as Ag thin films on plane substrates.

\section{METHODS}

\section{Materials}

Ag target (with a purity of $99.99 \%$ and size of $76 \times 5$ $\mathrm{mm}$, Advantage, Singapore), hydrochloric acid $(\mathrm{HCl}$, $36 \%$, Sigma-Aldrich), acetone (Sigma-Aldrich), double distilled water. All other chemicals were used as received without further purification.

\section{Preparation of Ag NPs film}

In this study, Ag nanostructured films were deposited at room temperature by using DC magnetron sputtering on the corning glass (size of $76 \times 26 \times 1 \mathrm{~mm}$, Marienfeld, Germany 900), and the base pressure was around $5 \times 10^{-5}$ Torr. First, the glass substrates were cleaned thoroughly in hydrochloric acid, acetone, then ultrasonicated in double distilled water in 15 min. Finally, substrates were dried under a stream of nitrogen. Before sputtering, argon plasma was used to etch the surface of the substrate for $10 \mathrm{~min}$ in order to remove residual particles on the substrate surface. The substrate holder was rotated at a speed of $5 \mathrm{rpm}$ during deposition. While the target was pre-sputtered for 5 min to dismiss contaminants and oxidized layers. The Ag target power was set at $9 \mathrm{~W}$, the Ar gas was used with a flow rate of $18 \mathrm{sccm}$, and the total pressure was approximately $2.5 \times 10^{-3}$ Torr. The amount of Ag was deposited with a constant time at 30 seconds, and the substrate temperatures are changed between $100^{\circ} \mathrm{C}$ and $400^{\circ} \mathrm{C}$. Moreover, the corresponding samples were denoted as Ag-y, where y was substrate temperatures.

\section{Characterization}

The structure and crystallinity of films were further investigated using X-ray diffraction measurements recorded using a Bruker D8 ADVANCE system with $\mathrm{CuK} \alpha$ radiation source $(\mathrm{l}=0.154056 \mathrm{~nm})$. The surface morphology of the films was observed using Hitachi S-4800 scanning electron microscopy (SEM, Hitachi S-4800) at room temperature, and atomic force microscopy (AFM, SPM 5500, USA). The optical properties of the films were characterized using a JASCOV670 spectrophotometer ranging from 300 to $800 \mathrm{~nm}$ scan rate of $200 \mathrm{~nm} \cdot \mathrm{min}^{-1}$ at room temperature.

\section{E. coli a ntibacterial experiment}

Bacterial culturing and plating were conducted following the standard methods described in previous research $^{18,19}$. The $1 \times 10^{6}$ colony-forming unit culture was allowed to drip on both o $n$ the surface of Corning glass containing the Ag NP film and in an unmodified glass slide (blank slide). They were then placed at room temperature. In the E.coli antibacterial performance test, all the experiments were conducted in a sterile environment. For all samples, the serial dilution was done, and the dilution was then spread uniformly on the surface into culture nutrient agar plates, and this plate was incubated at $37^{\circ} \mathrm{C}$ for 24 hours. The bactericidal activity of Ag NPs was investigated by the spread plate method. Finally, the number of colonies grown on the agar plates was counted and killing (\%) efficacy of Ag NPs was calculated using the following equation:

E.coli a ntibacterial efficacy $(\%)=\left[\left(\mathrm{N}_{\text {control }}-\right.\right.$ $\left.\left.\mathrm{N}_{\text {treated }}\right) / \mathrm{N}_{\text {control }}\right] \times 100$, where $\mathrm{N}_{\text {control }}, \mathrm{N}_{\text {treated }}$ are numbers of bacteria grown on the agar plates following treatment with glass and Ag NPs films, respectively.

\section{RESULTS}

The crystal structures of the Ag NPs films were characterized by X-ray diffraction patterns, and the results are shown in Figure 1. The diffraction peak appears at $2 \mathrm{q}=38.2$ and $44.4^{\circ}$ corresponding to lat- 
tice plane (111), (200) of silver crystal particle, respectively (JCPDS cards no 04-0783). As shown in Figure $1, t$ he XRD patterns are significantly changed at various substrate temperatures from $100^{\circ} \mathrm{C}$ to $400^{\circ} \mathrm{C}$. It is clearly observed that the peak intensity of crystal planes further enhances with increasing substrate temperature, which is mainly governed by improving in crystallinity of the face-c entered cubic phase of Ag. The preferred orientation of the Ag along with (111) and (200) plane is observed. Also, these peaks become more dominant at higher substrate temperatures; this might be associated with the thermodynamic phase boundary and surface interdiffusion phenomenon. While surface interdiffusion considered as the dominant kinetic process that plays a central role in controlling the crystalline morphology of the film. No typical diffraction peaks corresponding to silver oxide are observed, which maybe below the XRD detection limit ${ }^{20}$. Moreover, the (111) plane has a high atomic density of electrons that has more favorable for highly reactive ${ }^{21}$. Therefore, their antibacterial efficiency against $E$. Coli of Ag NPs is enhanced due to the interaction of the bacterial surface morphology with (111) plane.

The prepared samples are further evaluated by SEM to reveal the morphology characteristics of Ag NPs, and the results are shown in Figure 2.

As can be seen in Figure 2, when the substrate temperature increases, the particle size of $\mathrm{Ag}$ films increases leading to an increase in the mobility of silver atoms on the surface. This can be indicated that the distribution of Ag atoms is extremely in homogeneous that is governed by both local thermal energy and partially crystallizes the silver atoms. The particle size increases from 10 to 50 for substrate temperature at 100 to 400, respectively. Among all these types, the Ag-400 sample tends to the coalesce and form clusters, an enlargement of the clusters is linked to each other forming a film with a large open area fraction. With increasing the substrate temperature, surface characteristics such as number density, shape, size, inter-particle distance, and surface were adjusted through some scenarios ${ }^{22,23}$. At room temperature of growth, the nanoparticles are almost disordered that may be attributed to the streams of atoms based on moving and collision with each other on the surface. After a further $100^{\circ} \mathrm{C}$, atom movement is faster, this has resulted in the formation of single crystalline. After $200^{\circ} \mathrm{C}$ and above, the integration of atom clusters is almost completed with the rearrangement process during their progression to becoming more crystalline.
Moreover, the integration of many atom clusters into one governing structure can have a severe impact on the final structure of nanoparticles. This is attributed to the thermodynamic driving force that plays a central role in phase separation. However, the mechanism of phase separation is rather difficult to identify continuous or discontinuous transformation for these thin films, which was deposited at an elevated temperature. These have resulted in frozen in place by the incoming flux as reported by Adams et al. ${ }^{24}$. That can highly impact the antibacterial activity of $\mathrm{Ag}$ nanoparticles.

It is also interesting to consider whether Ostwald ripening processes, which are mainly governed by diffusion or coalescence of single atoms driven by a gradient in chemical potential causing the interchange of the neighboring atoms. This may also provide valuable insight into the growth of larger nanoparticle, more stable particles. As regards, the neighboring of two Ag NPs (the marked yellow circles denoted as Particle 1 and Particle 2) shows in Figure 2. Particle 1 reduces in size, whereas, particle 2 achieves a larger size after increasing temperature deposition for $400^{\circ} \mathrm{C}$, indicating that the particle appearance through an Ostwald ripening mechanism in this scenario ha s happened that forms a dense close-packed structure for $400^{\circ} \mathrm{C}$ as shown Figure 2d. These results indicate that this phenomenon plays a key role in the E. coli anti bacterial of Ag NPs films.

In order to understand further the role of increasing substrate temperature to the evolution of surface morphology, the root means square (RMS) roughness in Ag thin films was investigated by AFM analysis, as shown in Figures 3 and 4. The results have shown that the particle size increases with increasing substrate temperature. This can be attributed to the increased mobility of silver atoms due to the arising of local thermal energy lead to the formation of the disordered $\mathrm{Ag}$ phase $^{25}$. Moreover, the root means square and average grain size of Ag thin films increase with arising the substrate temperature, as shown in Figure 4e. The RMS values of Ag thin films at various substrate temperatures were $0.8,1.5,2.2,3.5$, and $6.0 \mathrm{~mm}$, respectively. Moreover, the surface appears rougher surface with more wrinkles (Figure 3d). Obviously, with increasing the substrate temperature from 100 to 400, the particle size and the RMS values increase, this is mainly attributed to the Ostwald ripening processes and the increment of energy surface at high temperature. In the case of Ostwald ripening, the higher surface to volume ratio and formation of bigger particles appeared due to coalescence. These are increased the particle size and inter particle distance ${ }^{26}$. In order to 


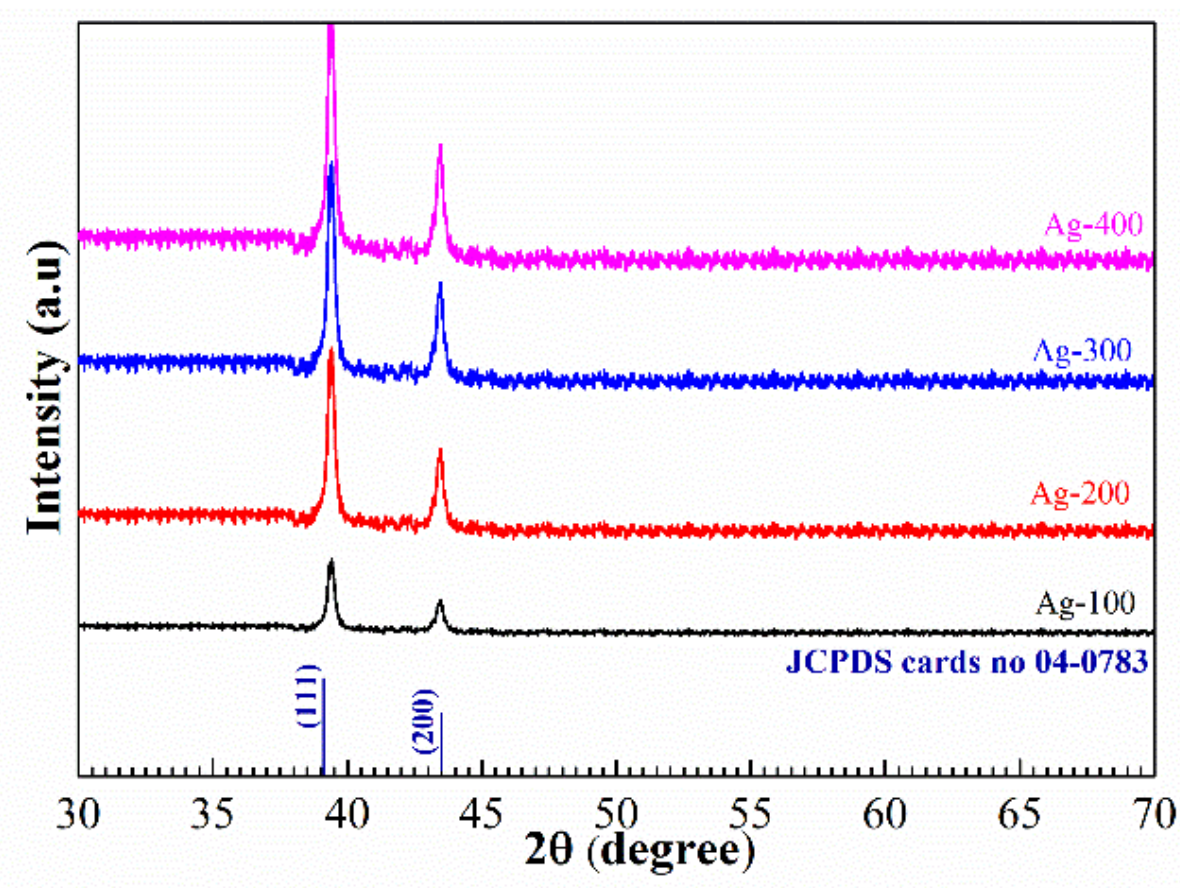

Figure 1: XRD patterns of Ag thin films at different substrate temperatures from $100^{\circ} \mathrm{C}$ to $400^{\circ} \mathrm{C}$.

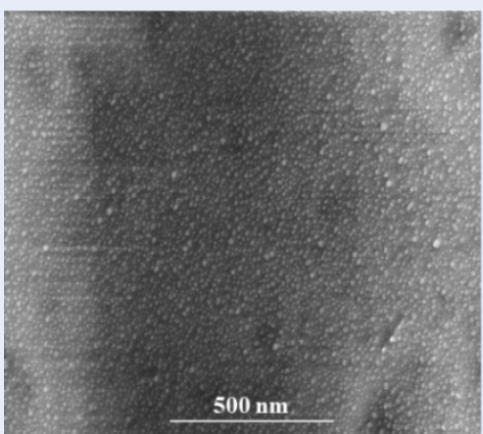

(a)

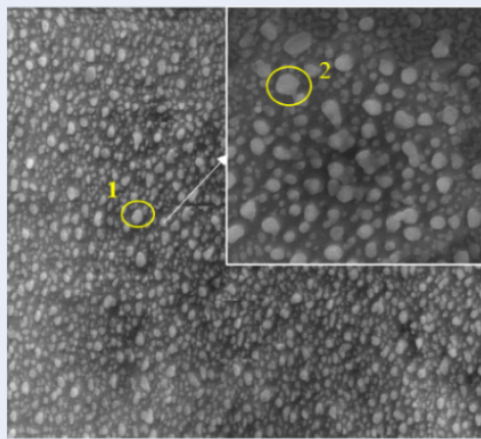

(c)

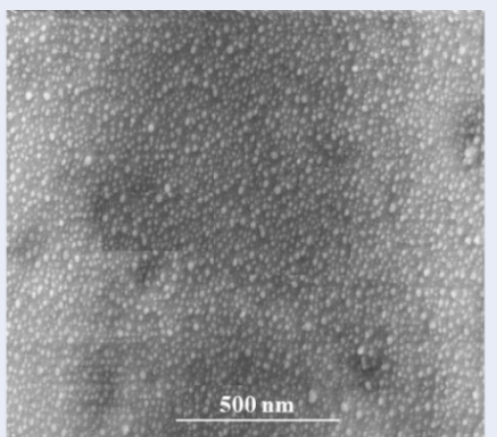

(b)

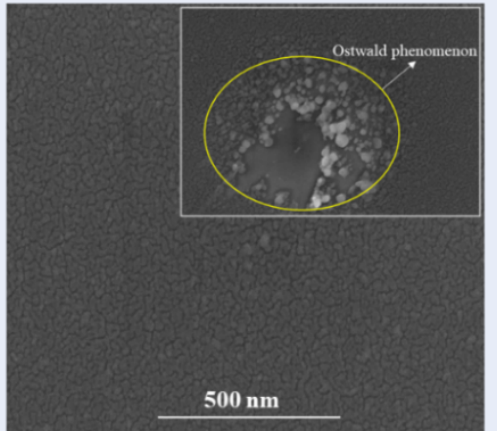

(d)

Figure 2: SEM images of substrate temperature of Ag NPs films at (a) $100^{\circ} \mathrm{C}$, (b) $200^{\circ} \mathrm{C}$, (c) $300^{\circ} \mathrm{C}$, and (d) $400^{\circ} \mathrm{C}$, respectively. 
better understand the mechanism of the growth $\mathrm{Ag}$ with increasing substrate temperature, a schematic of the growth in morphology is illustrated in Figure 5. In order to provide further information about its impact on optical properties based on their stage of morphological evolution, the UV-Visible absorption spectroscopy was used to evaluate the optical properties of samples. The absorption spectra of Ag NPs films at different substrate temperatures are clearly displayed in Figure 6. The variation of distribution in particle sizes of the Ag NPs film is attributed to the inhomogeneous broadening of the plasmon resonance peak. According to Mie theory ${ }^{18}$, the particle size decreased, the efficiency of absorption would be dominated over the scattering efficiency. Therefore, particles with a small size would be given rise to enhanced plasmon resonance. However, the distance between particles is large, so that coherent phase relations among the scattered light from different particles was not observed. Moreover, the red shift of the peak in the dipole resonance has been observed with increasing the particle size, which is assigned to the weakening of the restoring force. This can be explained by the increase of distance between charges on opposite sides of the particles, leading to a reduction in interaction. It is observed that the surface plasmon resonance (SPR) peak for Ag NPs films has a strong absorption at $450 \mathrm{~nm}$ corresponding to the dipolar resonance of Ag NPs. According to Figure 6 revealed that the Ag NPs film at various substrate temperatures shows a broadening and red shift of SPR spectra. At substrate temperatures $\geq 100^{\circ} \mathrm{C}$, the dramatic broadening along with pronounced red shift is obtained. This broadening can be attributed to the increase in particle size due to not much change in surface coverage, except crystalline form can be transformed. As a result, the optical properties of Ag nanostructures were dependent on their surface morphology through their growth stages.

It is well-known that silver has been considered as one of the most promising candidates to prevent infections for thousands of years. However, the silver-induced bactericidal effect is a complicated response related to the disruption of bacterial physiologies, such as the formation of disulfide bonds, iron metabolism, and homeostasis. So, in order to further evaluate the relationship between the particle size and toxicity of Ag NPs in eradicating Escherichia coli pathogenic, the live bacteria cells are treated with Ag NPs films at different substrate temperatures 100, 200, 300 , and $400^{\circ} \mathrm{C}$ respectively, as delineated Figure 7 .
These results indicate that the percentage of surviving bacteria reduces with increasing substrate temperature, suggesting that the antimicrobial efficacy increases while at $400^{\circ} \mathrm{C}$, the antimicrobial efficacy decreases. The substrate temperature plays a vital role in the $E$. coli antibacterial activity that is assigned to the significant change in the particle size and surface morphology of Ag NPs, as displayed in Figures 2, 3 and 4. In which, Ag-300 exhibits the highest the E. coli antimicrobial efficiency compared with others due to i) the high interaction between Ag NPs and E.coli bacteria based on the uniform distribution between the adjacent AgNPs, ii) the high toxicity path of Ag NPs based on the regular shapes and appropriate sizes of Ag NPS.

\section{DISCUSSION}

It is a fact that the increase of Ag NPs particle size is significantly reduced the specific surface area. Therefore, the release of dissolved Ag species is markedly retarded. As a result, their toxicity responses are lower. Therefore, it is essential to investigate the change in the particle size that may be caused by the dramatic change in toxicity and surface area of Ag NPs. Recent studies have reported that the highly efficient $E$. coli antibacterial activity of Ag NPs can be ascribed to the large surface area due to the interactions between the surface area of particles and microbial cells.

For instance, Morones et al. reported that the highly efficient antimicrobial performance of Ag NPs is due to the large surface area, which means that the percentage of the interaction of small particles was higher than larger particles of the same parent material ${ }^{19}$. Acharya et al. demonstrated that the distortion of the bacterial cell membrane by Ag nanosphere was higher than one-dimension nanostructure (Ag nanorod), which could mainly be governed by the granulate shape with a larger specific surface area as compared with 1-Dimension nanostructure (Ag nanorod) with low specific surface area ${ }^{27}$. Also, Hong et al. revealed that the nanosphere had a larger effective specific contact area, which means that they quickly achieved the interaction with bacterial cells and caused more damages $^{28}$. Thus, based on the above observations, it can be concluded that the specific surface area of Ag NPs plays a vital role in antimicrobial efficacy. Besides, it is clearly observed that Ag NPs with small particle size is shown to be more damaged as compared with larger particles in the silver-induced bactericidal because the smaller Ag NPS can be more easily penetrated E. coli bacteria than larger Ag NPs or AgNPs aggregates. This also substantiate $s$ the difference in the respective particle size as shown in SEM images (Figure 2). According to recent studies confirmed that the accumulation 


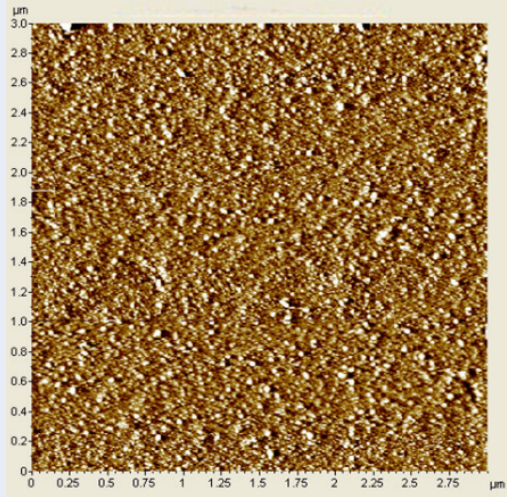

(a)

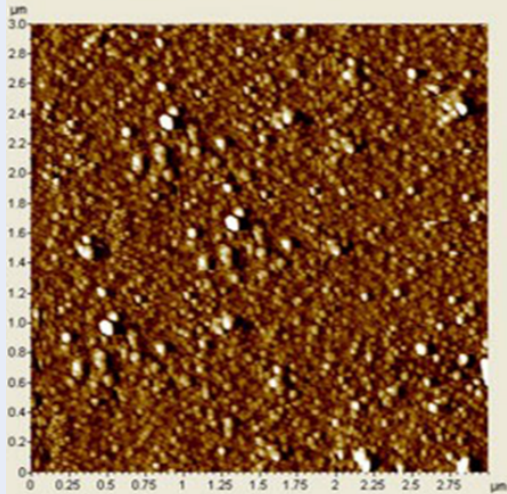

(c)

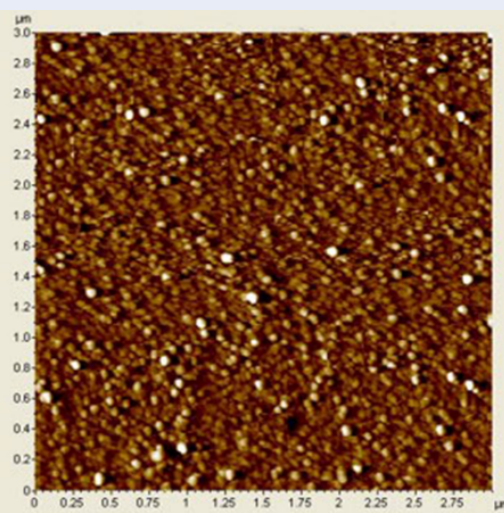

(b)

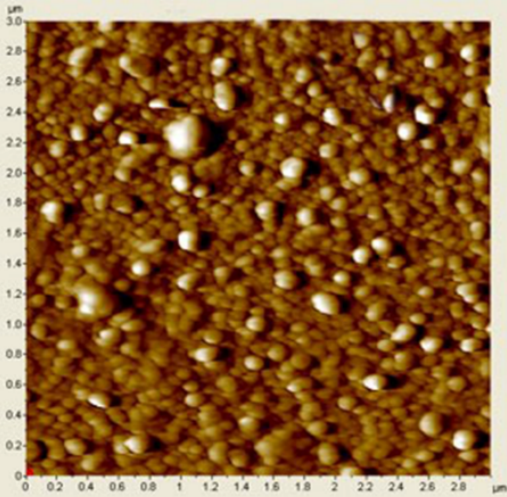

(d)

Figure 3: $2 \mathrm{D}$ AFM images of $\mathrm{Ag}$ thin films at various of substrate temperature (a) room temperature, (b) $100^{\circ} \mathrm{C},(\mathbf{c})$ $200^{\circ} \mathrm{C}$, (d) $300^{\circ} \mathrm{C}$, and $(\mathbf{e}) 400^{\circ} \mathrm{C}$.

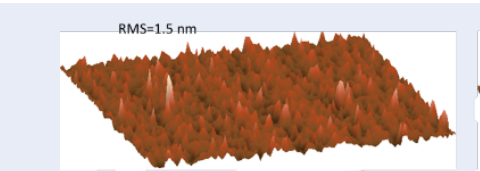

(a)

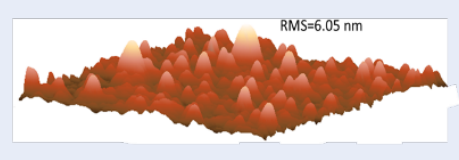

(d)

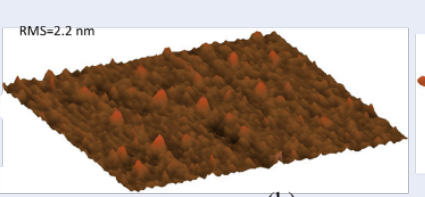

(b)

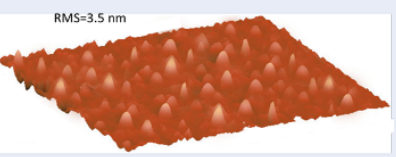

(c)

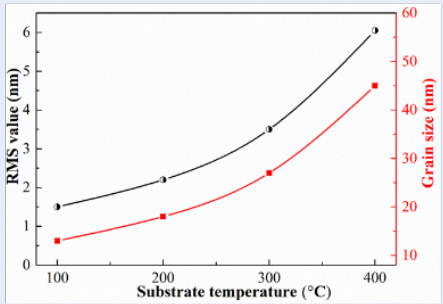

(e)

Figure 4: $3 \mathrm{D}$ AFM images of $\mathrm{Ag}$ thin film at various of substrate temperature (a) $100^{\circ} \mathrm{C}$, (b) $200^{\circ} \mathrm{C},(\mathbf{c}) 300^{\circ} \mathrm{C}$, and (d) $400^{\circ} \mathrm{C}$. (e) RMS values and average grain size of Ag thin films at different substrate temperatures. 

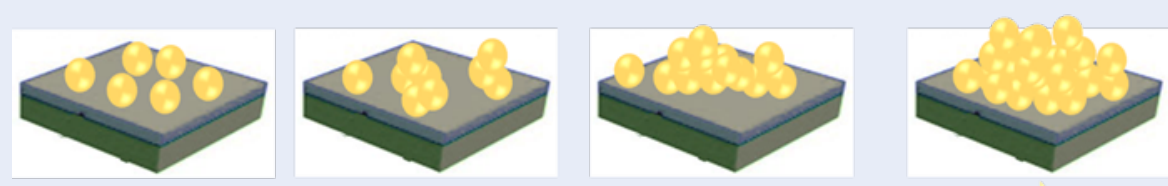

Increasing substrate temperature

Figure 5: Schematic of the growth in morphology as a result of increasing substrate temperature.

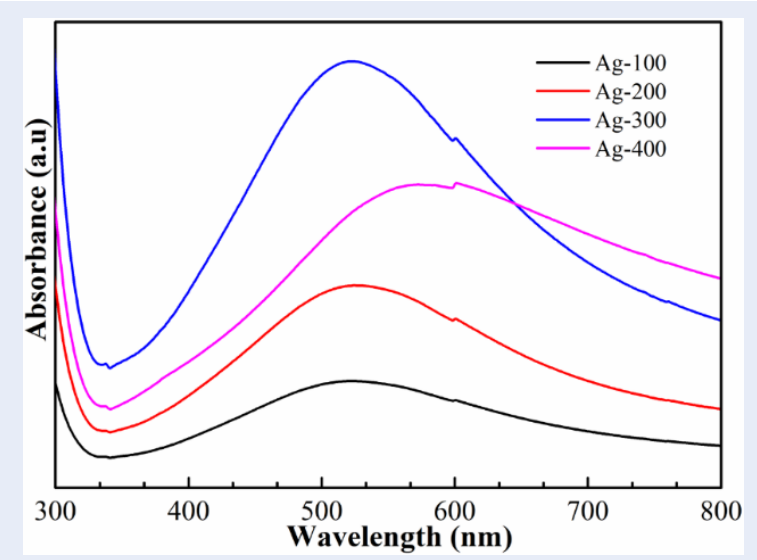

Figure 6: UV-vis spectra of different annealing temperatures from $100^{\circ} \mathrm{C}$ to $400^{\circ} \mathrm{C}$ of $\mathrm{Ag} \mathrm{NPs}$ films.

of different sizes of Ag NPs in the food chain was investigated the toxicity of $\mathrm{AgNPs}$ and $\mathrm{Ag}^{+}$ions during the antimicrobial process. The results revealed that some of the small Ag NPs remained in the digestive lumen, subcutaneous tissue, and gonad. This verified that the Ag NPs uptake at the intracellular level and only small particles or partial aggregation of silver nanoparticles were detected in the cells, whereas larger aggregation was not internalized in . coli $^{29-31}$. On the other hand, the antibacterial efficacy of $\mathrm{Ag}$ NPs films is affected by controlling the toxicity path of Ag NPs based on changing the particle size. The small Ag NPs induced more cellular toxicity than larger particles. For example, Liu et al. reported that Ag NPs with size particle of $5 \mathrm{~nm}$ had more toxicity than 20 and $50 \mathrm{~nm} \mathrm{Ag} \mathrm{NPs} \mathrm{towards} \mathrm{human} \mathrm{cells}{ }^{32}$. Wang et al. reported that Ag NPs nanoparticle size of $10 \mathrm{~nm}$ was much more cytotoxic than 40 and $75 \mathrm{~nm} \mathrm{Ag} \mathrm{NPs}$ to human lung cells. However, toxicity between 10 $\mathrm{nm}$ citrate and $10 \mathrm{~nm}$ PVP-coated Ag NPs was not observed $^{33}$. These results are well justified deduce that the Ag NPs toxicity related to their antimicrobial efficiency not only dependent particle size but also based on bacterial type. The toxicity plays a significant role in the antimicrobial capability of Ag NPs, which is attributed to the release of $\mathrm{Ag}^{+}$cations that can be interacted with cells and intracellular macromolecules such as proteins and DNA. Besides, an accumulation of intercellular reactive oxygen species (ROS) reacts directly with protein and causes oxidative stress. So, the partial or permanent loss of structure and/or function of the cellular protein may be caused by the above processes leads to the bacterial growth is inhibited. On the other side, the Ag NPs shows excellent $E$. coli antibacterial effect at different substrate temperature due to the presence of (111) plane. This can be attributed to the plane contains a high atomic density of electrons. Overall, our results showcased that $\mathrm{Ag}$ NPs films can inhibit the growth of bacteria based on the density distribution and particle size of Ag NPs leading to the change of their toxicity.

\section{CONCLUSIONS}

In summary, we have investigated the antimicrobial activity of Ag NPs films prepared by DC magnetron sputtering technique. Their optical properties were analyzed by UV-Vis spectroscopy, while, the structure and morphological properties were investigated by XRD, FE-SEM, and AFM, respectively. Besides, 


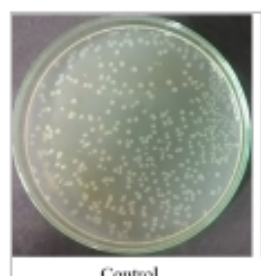

Control

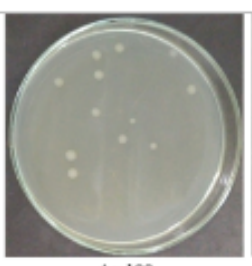

Az- -100
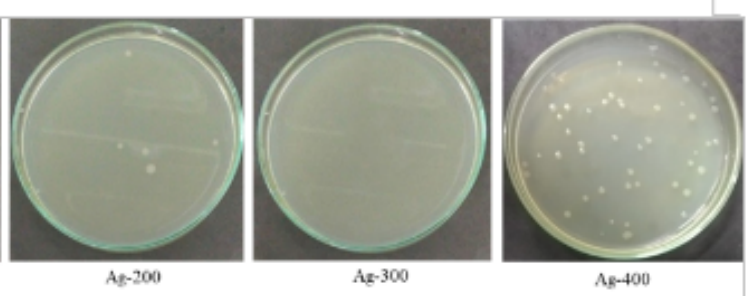

(a)

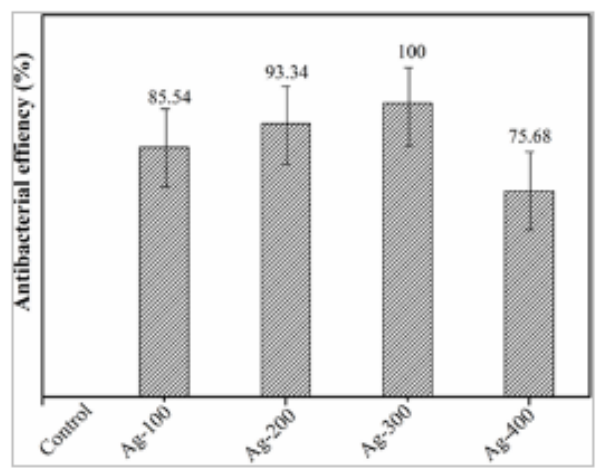

(b)

Figure 7: Development of $E$. coli colonies (a) and $E$. coli antibacterial activity of different substrate temperatures of Ag NPs films against E. coli calculated by plate count method (b).

their antimicrobial efficacy is tuned by adjusting the change of morphology of Ag NPs, such as density distribution and particle size. The results show that the E. coli anti-bacterial efficacy of the Ag-300 sample could reach up to $\sim 100 \%$. This can be attributed to the change in the particle size and surface morphology of Ag NPs leading to the change of the Ag NPs toxicity. The work also provides a better understanding of the effect of Ag NPs morphology in enhancing the surface plasmon resonance related to Ostwald ripening processes that can be developed more efficient desired antimicrobial systems under the visible regime in the future.

\section{ABBREVIATIONS}

NPs: Nanoparticles

Ag NPs: Silver nanoparticles

\section{COMPETING INTERESTS}

The authors declare that there is no conflict of interest regarding the publication of this article.

\section{AUTHORS' CONTRIBUTIONS}

Ton Nu Quynh Trang has conceived of the present idea, carried out and written the manuscript with support from Vu Thi Hanh Thu. Ton Nu Quynh Trang and Le Thi Ngoc Tu carried out the experiments in group. Tran Van Man has supported the analysis techniques.

\section{ACKNOWLEDGMENTS}

This research is funded by the University of Science, VNU-HCM, under grant number T2019-12.

\section{REFERENCES}

1. Boisselier E, Astruc D. Gold nanoparticles in nanomedicine: preparations, imaging, diagnostics, therapies and toxicity. Chemical society reviews. 2009;38(6):1759-82.

2. Liu CJ, Burghaus U, Besenbacher F, Wang ZL. Preparation and characterization of nanomaterials for sustainable energy production. ACS Nano. 2010;4:5517-5526.

3. Seil JT, Webster TJ. Antimicrobial applications of nanotechnology: methods and literature. International journal of nanomedicine. 2012;7:2767-81.

4. Tolaymat TM, ElBadawy AM, Genaidy A, Scheckel KG, Luxton TP, Suidan M. An evidence-based environmental perspective of manufactured silver nanoparticle in syntheses and applications: a systematic review and critical appraisal of peerreviewed scientific papers. Science of the total environment. 2010 Feb 1;408(5):999-1006.

5. Weber DJ, Anderson D, Rutala WA. The role of the surface environment in healthcare-associated infections. Current opinion in infectious diseases. 2013 Aug 1;26(4):338-382.

6. Vukomanović M, Žunič V, Š Kunej, Jančar B, Jeverica S, Suvorov D. Nano-engineering the antimicrobial spectrum of lantibiotics: activity of nisin against gram negative bacteria. Scientific reports. 2017 Jun 28;7(1):43241-13. 
7. Seil JT, Webster TJ. Antimicrobial applications of nanotechnology: methods and literature. International journal of nanomedicine. 2012;7:2767-81.

8. Chaloupka K, Malam Y, Seifalian AM. Nanosilver as a new generation of nanoproduct in biomedical applications. Trends in biotechnology. 2010 Nov 1;28(11):580-8.

9. Sharma VK, Filip J, Zboril R, Varma RS. Natural inorganic nanoparticles-formation, fate, and toxicity in the environment. Chemical Society Reviews. 2015;44(23):8410-23.

10. Xiu ZM, Zhang QB, Puppala HL, Colvin VL, Alvarez PJ. Negligible particle-specific antibacterial activity of silver nanoparticles. Nano letters. 2012 Jul 9;12(8):4271-5.

11. Acharya D, Singha KM, Pandey $P$, Mohanta B, Rajkumari J, Singha LP. Shape dependent physical mutilation and lethal effects of silver nanoparticles on bacteria. Scientific reports. 2018 Jan 9;8(1):201-11.

12. Ding D, Chen L, Dong S, Cai H, Chen J, Jiang C, et al. Natural ageing process accelerates the release of $\mathrm{Ag}$ from functional textile in various exposure scenarios. Scientific reports. 2016 Nov 21;6:373141-8.

13. Nam SH, Kwak Jl, An YJ. Quantification of silver nanoparticle toxicity to algae in soil via photosynthetic and flowcytometric analyses. Scientific reports. 2018 Jan 10;8(1):292112.

14. Luo $X, X u$ S, Yang $Y$, Li L, Chen S, Xu A, et al. Insights into the ecotoxicity of silver nanoparticles transferred from Escherichia coli to Caenorhabditis elegans. Scientific reports. 2016 Nov 4;6:364651-12.

15. Polte J. Fundamental growth principles of colloidal metal nanoparticles-a new perspective. CrystEngComm. 2015;17(36):6809-30.

16. Koziej D. Revealing Complexity of Nanoparticle Synthesis in Solution by in Situ Hard X-ray Spectroscopy-Today and Beyond. Chemistry of Materials. 2016 Apr 8;28(8):2478-90.

17. Taglietti A, Arciola CR, D'Agostino A, Dacarro G, Montanaro L, Campoccia $D$, et al. Antibiofilm activity of a monolayer of silver nanoparticles anchored to an amino-silanized glass surface. Biomaterials. 2014 Feb 1;35(6):1779-88.

18. Born M, Wolf E. Principles of optics: electromagnetic theory of propagation, interference and diffraction of light. Elsevier; 2013 Jun 1.

19. Morones JR, Elechiguerra JL, Camacho A, Holt K, Kouri JB, Ramrez JT, et al. The bactericidal effect of silver nanoparticles. Nanotechnology. 2005 Aug 26;16(10):2346-53.

20. Devi LG, Kavitha R, Nagaraj B. Bulk and surface modification of $\mathrm{TiO} 2$ with sulfur and silver: Synergetic effects of dual surface modification in the enhancement of photocatalytic activity. Materials Science in Semiconductor Processing. 2015 Dec 1;40:832-9.

21. Periasamy $S$, Joo HS, Duong AC, Bach TH, Tan VY, Chatterjee SS, et al. How Staphylococcus aureus biofilms develop their characteristic structure. Proceedings of the National Academy of Sciences. 2012 Jan 24;109(4):1281-6.

22. Challa SR, Delariva AT, Hansen TW, Helveg S, Sehested J, Hansen PL, et al. Relating rates of catalyst sintering to the disappearance of individual nanoparticles during Ostwald ripening. Journal of the american chemical society. $2011 \mathrm{Dec}$ 1;133(51):20672-5.

23. Hejral U, Mller P, Balmes O, Pontoni D, Stierle A. Tracking the shape-dependent sintering of platinum-rhodium model catalysts under operando conditions. Nature communications. 2009 Mar 9;7:109641-8.

24. Adams CD, Atzmon M, Cheng YT, Srolovitz DJ. Transition from lateral to transverse phase separation during film codeposition. Applied physics letters. 1991 Nov 11;59(20):25357.

25. Mohan D, Sunandana CS. lodization of rf sputter induced disordered Ag thin films reveals volume plasmon-exciton "transition". Journal of applied physics. 2006 Sep 15;100(6):64314110.

26. Ghosh T, Karmakar P, Satpati B. Electrochemical Ostwald ripening and surface diffusion in the galvanic displacement reaction: control over particle growth. RSC Advances. 2015;5(114):94380-7.

27. Acharya D, Singha KM, Pandey P, Mohanta B, Rajkumari J, Singha LP. Shape dependent physical mutilation and lethal effects of silver nanoparticles on bacteria. Scientific reports. 2018 Jan 9;8(1):201-11.

28. Hong $X$, Wen J, Xiong $X$, Hu Y. Shape effect on the antibacterial activity of silver nanoparticles synthesized via a microwaveassisted method. Environmental science and pollution research. 2016 Mar 1;23(5):4489-97.

29. Luo $X, X u$ S, Yang Y, Li L, Chen S, Xu A, et al. Insights into the ecotoxicity of silver nanoparticles transferred from Escherichia coli to Caenorhabditis elegans. Scientific reports. 2016 Nov 4;6:364651-12.

30. Masoud EA, Al-Hajry AM, Al-Marrani A. Antibacterial activity of silver nanoparticles synthesized by sidr (Ziziphus spinaChristi) leaf extract against pathogenic bacteria. Int J Curr Microbiol App Sci. 2016;5:226-36.

31. Beyene HD, Werkneh AA, Bezabh HK, Ambaye TG. Synthesis paradigm and applications of silver nanoparticles (AgNPs), a review. Sustainable materials and technologies. $2017 \mathrm{Sep}$ 1;13:18-23.

32. Liu W, Wu Y, Wang C, Li HC, Wang $\mathrm{T}$, Liao CY, et al. Impact of silver nanoparticles on human cells: effect of particle size. Nanotoxicology. 2010 Sep 1;4(3):319-30.

33. Gliga AR, Skoglund S, Wallinder IO, Fadeel B, Karlsson HL. Sizedependent cytotoxicity of silver nanoparticles in human lung cells: the role of cellular uptake, agglomeration and Ag release. Particle and fibre toxicology. 2014 Dec;11(1):2-17. 